\title{
Interaction Energy between an Atomic Force Microscope Tip and a Charged Particle in Electrolyte
}

\author{
Wai-Ting Lam, Fredy R. Zypman \\ Yeshiva University, New York, NY, USA \\ Email: zypman@yu.edu
}

How to cite this paper: Lam, W.-T. and Zypman, F.R. (2016) Interaction Energy between an Atomic Force Microscope Tip and a Charged Particle in Electrolyte. Journal of Applied Mathematics and Physics, 4, 1989-1997.

http://dx.doi.org/10.4236/jamp.2016.411199

Received: October 18, 2016

Accepted: November 7, 2016

Published: November 10, 2016

Copyright $\odot 2016$ by authors and Scientific Research Publishing Inc. This work is licensed under the Creative Commons Attribution International License (CC BY 4.0).

http://creativecommons.org/licenses/by/4.0/

\begin{abstract}
A variational principle to the nonlinear Poisson-Boltzmann equation $(\mathrm{PB})$ in three dimensions is used to first obtain solutions to the electrostatic potential surrounding a pair of spherical colloidal particles, one of them modeling the tip of an Atomic Force Microscope. Specifically, we consider the PB action integral for the electrostatic potential produced by charged colloidal particles and propose an analytical ansatz solution. This solution introduces the density and its corresponding electrostatic potential parametrically. The PB action is then minimized with respect to the parameter. Polynomial-exponential approximations for the parameters as functions of tipparticle separation and boundary electrostatic potential are obtained. With that information, tip-particle energy-separation curves are computed as well. Finally, based on the shape of the energy-separation curves, we study the stability properties predicted by this theory.
\end{abstract}

\section{Keywords}

Atomic Force Microscopy, Colloids, Electrostatics in Liquids

\section{Introduction}

An open problem of current scientific and technological interest is the theoretical prediction of the force between an Atomic Force Microscope (AFM) probe and a charged particle, in particular when both are immersed in an electrolytic environment [1]. The main interest of this problem comes from the need to understand the electrostatics of biological matter, a problem in which water is inherently present [2]. In addition, AFM has become the de facto metrological tool to probe organic and inorganic matter from the micron down to the nanometer length scales [3]. The tip comprises the sensing 
element of the AFM and, at its apex ranges in size from microns to nanometers, thus the ability of the AFM to probe those length-scales. When the AFM tip is immersed in an electrolyte, it can gain surface charge due to $\mathrm{pH}$, and also can develop a diffuse charge layer due to the presence of ions in solution [4]. Thus we see a natural conceptual connection between AFM measurements in liquid and colloidal science, whereby the interest is in the interaction between colloidal particles and their corresponding stability. Therefore, although our interest is in AFM in liquid, the results obtained here are readily usable in colloidal systems. We focus here in a liquid system in which 1$1000 \mathrm{~nm}$ particles are submerged in an ionic solution. Colloidal systems comprise one of the primary types of mixtures in chemistry. One of the central problems in colloids is their stability [5], that is, under known conditions, such as concentration; will the system coagulate or remain indefinitely stable?

The stability of colloids is indeed known to depend on the presence of charge at their surfaces [6]; the electrical double layer controls electrostatic stabilization. When particles approach each other, the interaction leads to the rearrangement of charges in the ambient liquid, outside of the colloidal particles. For instance, these interactions could be determined by the surface charge on the particles and electrolyte concentration. The stability of colloidal systems is interesting conceptually, and critical for industrial applications. In chemistry, there are different types of colloidal systems such as solid-liquid dispersions (suspensions), liquid-liquid dispersions (emulsions) and gas-liquid dispersions (foams). Paints, milk, proteins as well as fog are some of the daily examples of colloids [7] [8].

Mathematically, the stability depends on the details of the pair-wise energy as a function of separation of colloidal particles [9]. The valleys of such function determine the separations of the possible equilibrium. One approach to obtain that energy is to first solve the Poisson-Boltzmann (PB) equation, whose solution gives the charge density and electrostatic potential in the liquid surrounding the colloidal particles [10]. In general, PB equation provides the distribution of the electric potential in solution with charged ions present. This distribution, in turn, provides information to determine how the electrostatic interactions will affect colloidal forces. PB is a nonlinear second-order partial differential equation which has an exact known solution only in one dimension. In higher dimensions, $\mathrm{PB}$ equation is commonly solved by numerical analysis. Alternatively, if the colloidal particle charge or voltage is not high, the PB equation can be linearized, in which case solutions for spherical [11] and cylindrical [12] geometries have also been obtained. For the particular geometry of sphere-plane, useful to study charge transfer in scanning tunneling microscopy and forces in atomic force microscopy, there have been analytical and numerical approaches to the nonlinear PB equation in three dimensions [13] [14] [15] [16]. Here, we present a method to tackle the full nonlinear PB equation in three-dimensions for interacting particles. The method is analytical, based on the choice of a parametric trial family of functions. While the method is approximate, its analytical nature should provide conceptual insight into the problem.

The exact $\mathrm{PB}$ nonlinear equation in $3 \mathrm{D}$ is not amenable to analytical solutions even 
for a single colloidal particle in the electrolyte. We here consider the problem of two interacting particles by introducing an ansatz for the charge density function and corresponding electrostatic potential parametrically; the variational method is then used to minimize the PB functional with respect to the parameters.

\section{Electrostatics Potential Produced by a Pair of Colloids}

Figure 1 shows schematics of the system of interest. Two charged spherical colloidal particles of unit diameter are separated by a distance $d$.

The PB equation is typically obtained by combining Poisson's equation [17] and the Boltzmann factor [18] for the distribution of electrostatic energies at a given temperature. Thus Poisson's equation gives the relationship between the electrical potential $\Phi(\boldsymbol{R})$ and the charge density $\rho(\boldsymbol{R})$ at location $\boldsymbol{R}$,

$$
\nabla^{2} \Phi(\boldsymbol{R})=-(4 \pi / \epsilon) \rho(\boldsymbol{R})
$$

with $\epsilon$ is the dielectric constant of the surrounding fluid. On introducing the Boltzmann distribution of ions, the non-linear PB equation is obtained,

$$
\nabla^{2} \Phi(\boldsymbol{R})=-(8 \pi n e / \epsilon) \sinh \left(e \Phi(\boldsymbol{R}) / k_{B} T\right)
$$

where $n$ is the ion bulk concentration of electrolyte, $T$ is the absolute temperature, $e$ the ion charge magnitude of anions and cations, and $k_{B}$ is Boltzmann's constant.

Equation (2) is converted into dimensionless form [2] by defining the dimensionless electrostatic potential $\varphi(\boldsymbol{r})=\frac{e}{k_{B} T} \Phi(\boldsymbol{R})$ and the dimensionless position vector $\boldsymbol{r}=\sqrt{\frac{8 \pi n e^{2}}{k_{B} T \epsilon}} \boldsymbol{R}$,

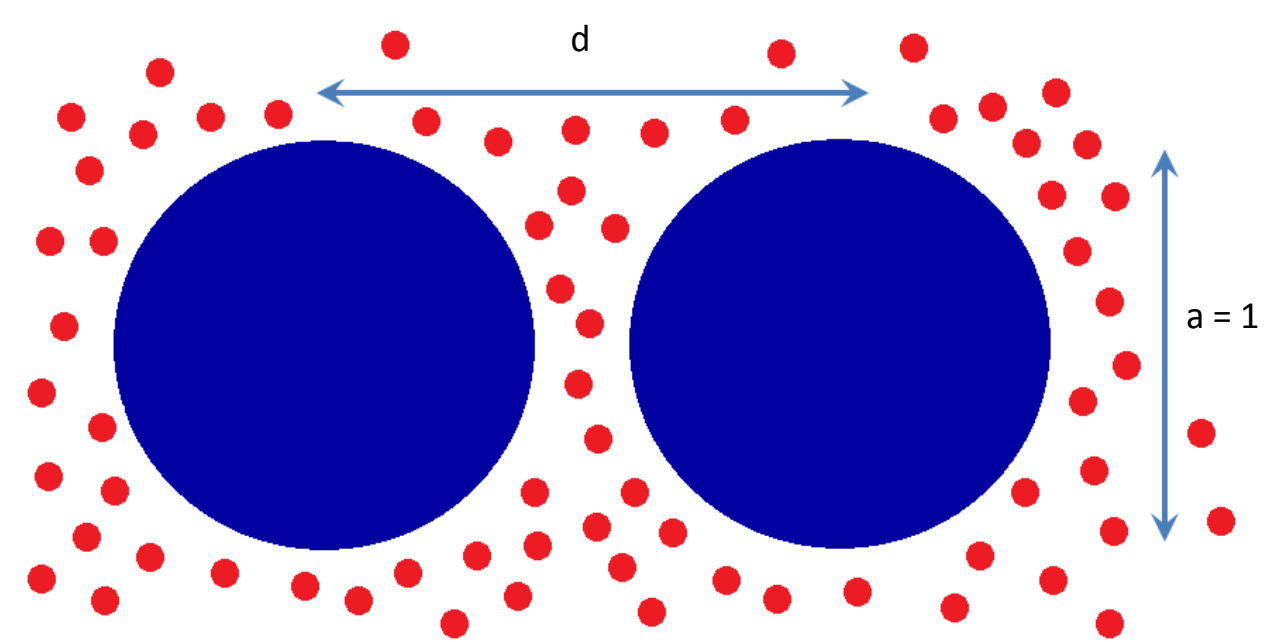

Figure 1. Two colloidal particles (large, blue) separated by a distance $d$. The unit of length throughout the paper is the particles diameter, or the AFM tip diameter. The small red particles represent the ions dissolved in water and are treated as a continuum in the Poisson-Boltzmann approach. These ions could be different, we show them here with the same color for graphical simplicity. 


$$
\nabla^{2} \varphi=-\sinh \varphi
$$

where $\varphi$ now represents the dimensionless electrostatic potential, and the Laplacian in Equation (3) is with respect to $\boldsymbol{r}$. Since $n$ has unites of inverse volume, and $\epsilon$ is the absolute dielectric constant, $\frac{8 \pi n e^{2}}{k_{B} T \epsilon}$ has units of inverse area.

Equation (3) can be derived from a variational principle, by applying Euler-Lagrange to the action

$$
I=\int_{\text {Space }}\left[\frac{1}{2}|\nabla \varphi|^{2}+\cosh (\varphi)-1\right] \mathrm{d} V
$$

where $V$ is volume. The minimum of I occurs for the function $\varphi$ that satisfies the Euler-Lagrange equation, which gives rise Equation (3).

Taking $z$ as the axis that joins the centers of the two colloidal particles, and due to the axial symmetry of the problem, we rewrite the action in cylindrical coordinates as

$$
4 \pi \int_{0}^{\infty} \int_{0}^{\infty}\left[\frac{1}{2}|\nabla \varphi|^{2}+\cosh (\varphi)-1\right] \eta \mathrm{d} \eta \mathrm{d} z
$$

where $\eta$ is the radial polar coordinate in the xy plane, while the angular polar integration is readily performed and gives $2 \pi$. The additional factor of 2 comes from integrating $z$ in half space and multiplying by 2 due to mirror symmetry. To make progress, we propose the following ansatz for the density and corresponding electrostatic potential which depends on the parameter $k$,

$$
\varphi(\eta, z)=\varphi_{0} \exp \left\{-\frac{k}{2}\left[\sqrt{\left(z-\frac{d}{2}\right)^{2}+\eta^{2}}-\frac{1}{2}\right]\left[\sqrt{\left(z+\frac{d}{2}\right)^{2}+\eta^{2}}-\frac{1}{2}\right]\right\}
$$

where $\varphi_{0}$ is the Dirichlet boundary condition, $d$ is the center-to-center separation between the two spherical colloids and $k$ is a constant that can be interpreted as an inverse Debye length times the radius of the interacting particles. The intuitive justifications for the functional form are 1) its exponential decay typical of ionic screening, 2) that proper boundary conditions $\varphi(\eta, z)=\varphi_{0}$ are achieved at the surface of the colloids, and 3) that as $d \rightarrow \infty$, the electrostatic potential between the two colloids tends to zero. Figure 2 shows a contour plot of the potential around the colloidal particles for a choice of $k$.

To emphasize, this choice of potential is dictated first by the fact that the potential must rapidly approach its bulk value away from the spheres, and second by the fact that the electrostatic potential must have a constant value at the surface of the colloidal particles (Dirichlet boundary conditions).

To find the sought solution to the original PB equation (Equation (3)), we minimize the $\mathrm{PB}$ action functional with respect to the parameter $k$. For fixed potential $\varphi_{0}$ and fixed separations $d$, we find the constant $k$ (i.e. minimum point $k=k\left(\varphi_{0}, d\right)$ ) for the proposed $\varphi(x, y)$ that minimizes the action.

As Figure 3 shows, for small separations, the data suggests that there is an approximately linear relationship between the best constant $k_{\text {best }}$, and the separation $d$ for each 


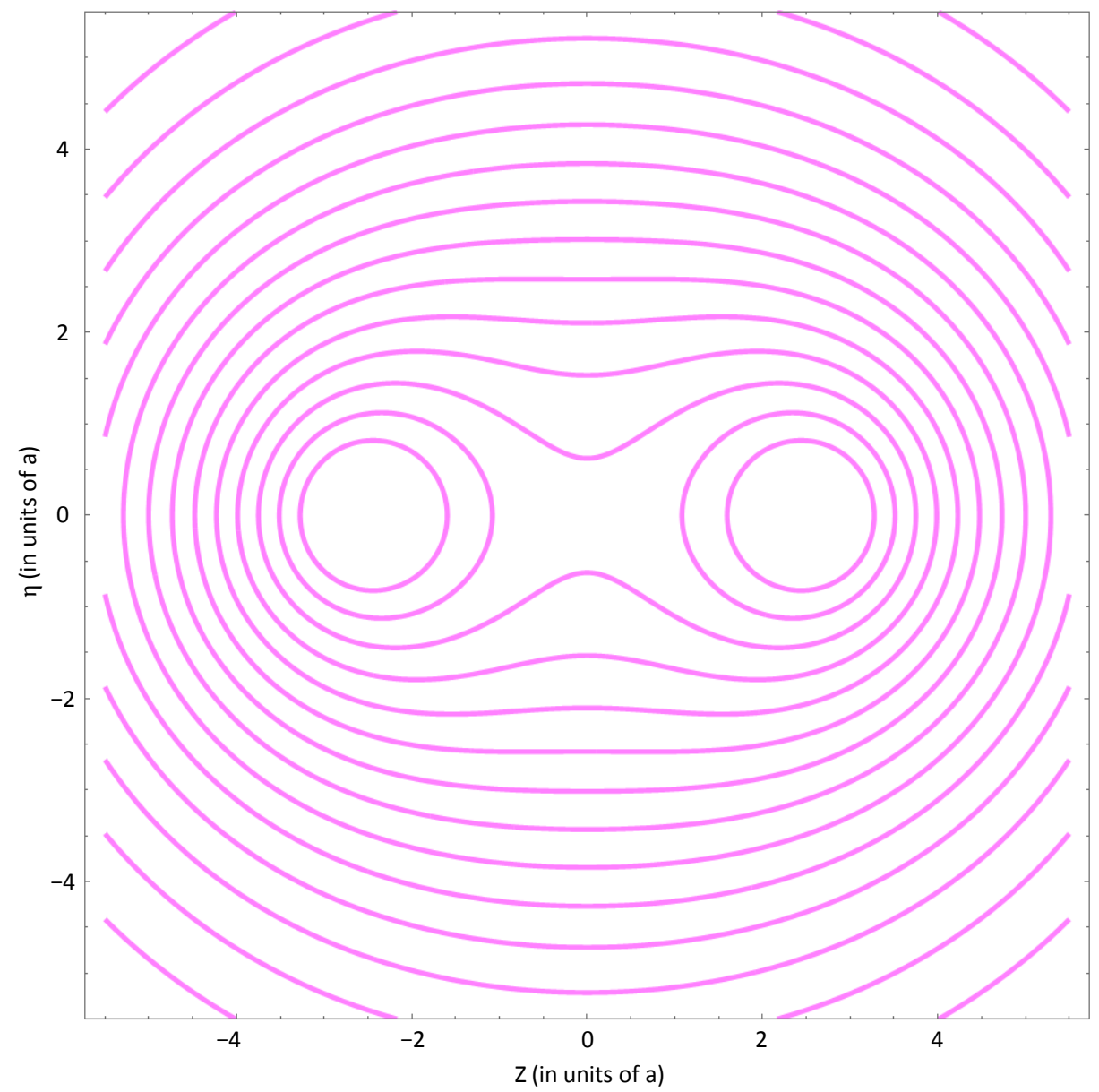

Figure 2. Contour plot of $\varphi(\eta, z)$. The potential is constant $\varphi_{0}$ at the surfaces of the particles, becomes spherical far away while decaying to zero.

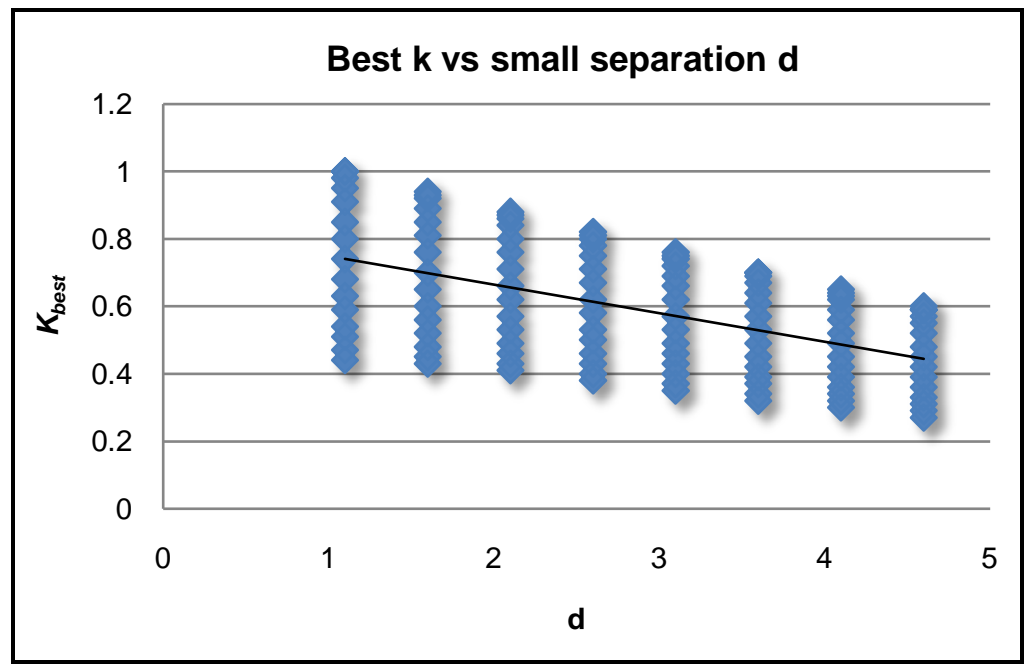

Figure 3. Graphs of $k_{\text {best }}$ as a function of small separation $d$, as $\varphi_{0}$ is changed. It shows an approximately linear relationship between $k_{\text {best }}$ and small separation $d$ for each $\varphi_{0}$. The black line is drawn to show the average trend between $k_{\text {best }}$ and $\varphi_{0}$. 
boundary condition $\varphi_{0}$. It leads us to further investigate the relationship between the linear relationship and the boundary condition $\varphi_{0}$.

We obtain polynomial approximations for the functions that relate the linear parameters (that are the slopes and $\eta$-intercepts) and the boundary conditions (as shown in Figure 4(a) and Figure 4(b)).

Moreover, for large separations between the colloidal particles, the best constant $k$ converges to 0.1 for all of the boundary conditions $\varphi_{0}$. This should be a universal feature regardless of the model used since at large separations we should obtain a simple superposition the potential around single spheres.

The functional forms for the $k_{\text {best }}$ allow us to write a simple function as follows (Figure 5).

$$
k_{\text {best }}=(A(\varphi)-0.1) \mathrm{e}^{\frac{B(\varphi)}{A(\varphi)-0.1}+0.1}
$$

where $A(\varphi)$ is the polynomial approximation between the linear parameter- $\eta$-intercepts and $\varphi_{0}, B(\varphi)$ is the polynomial approximation between the other linear parameter-slope and $\varphi_{0}$, and $d$ is the center-to-center separation between the colloids.

\section{Colloid Interaction Energy}

Since the charge is distributed in the whole space that surrounds the colloidal particles, we have the energy as a function of separation $d[13]$

$$
E_{\varphi}(d)=\frac{1}{2} \int_{\text {space }} \mathrm{d} \boldsymbol{r} \rho_{\varphi}(d) V_{\varphi}(d)
$$

where to recall $\rho$ is density and $\varphi$ is voltage, which are now known from the previous section. For each boundary condition, the integral in (8) is performed for the corresponding optimal value of $k$.

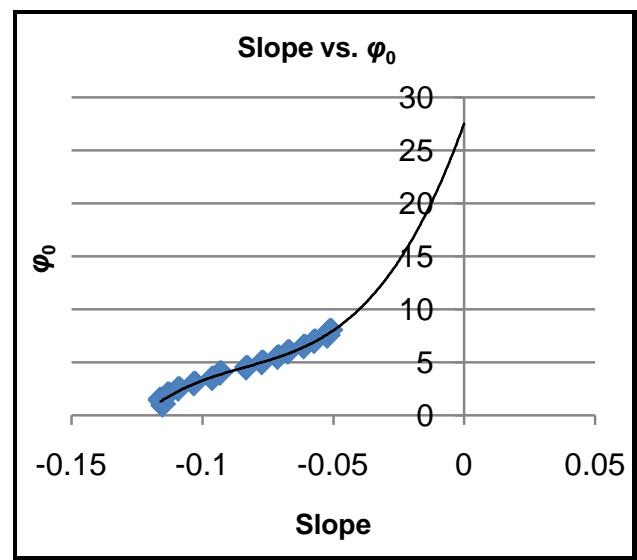

(a)

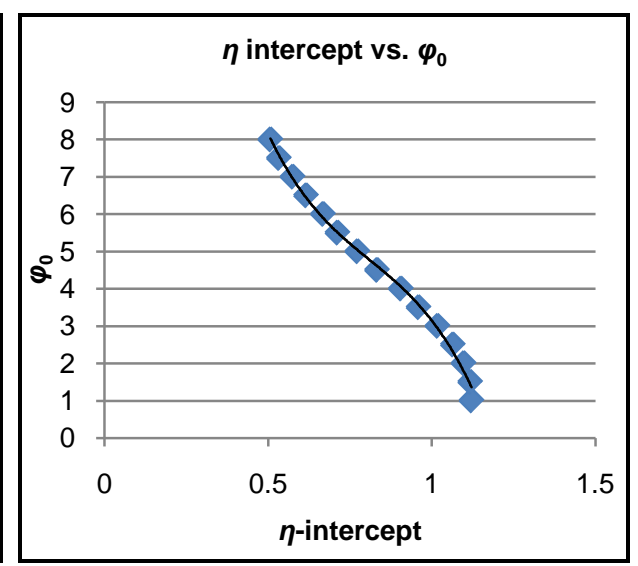

(b)

Figure 4. (a) Polynomial approximation between the linear parameter-slope and the boundary conditions $\varphi_{0}$. This is the slope of $k_{\text {best }}$ vs $d$ (Figure 3). (b) Polynomial approximation between the $\eta$-intercept and the boundary condition $\varphi_{0}$.This is the $\eta$-intercept of $k_{\text {best }}$ vs $d$ (Figure 3 ). 


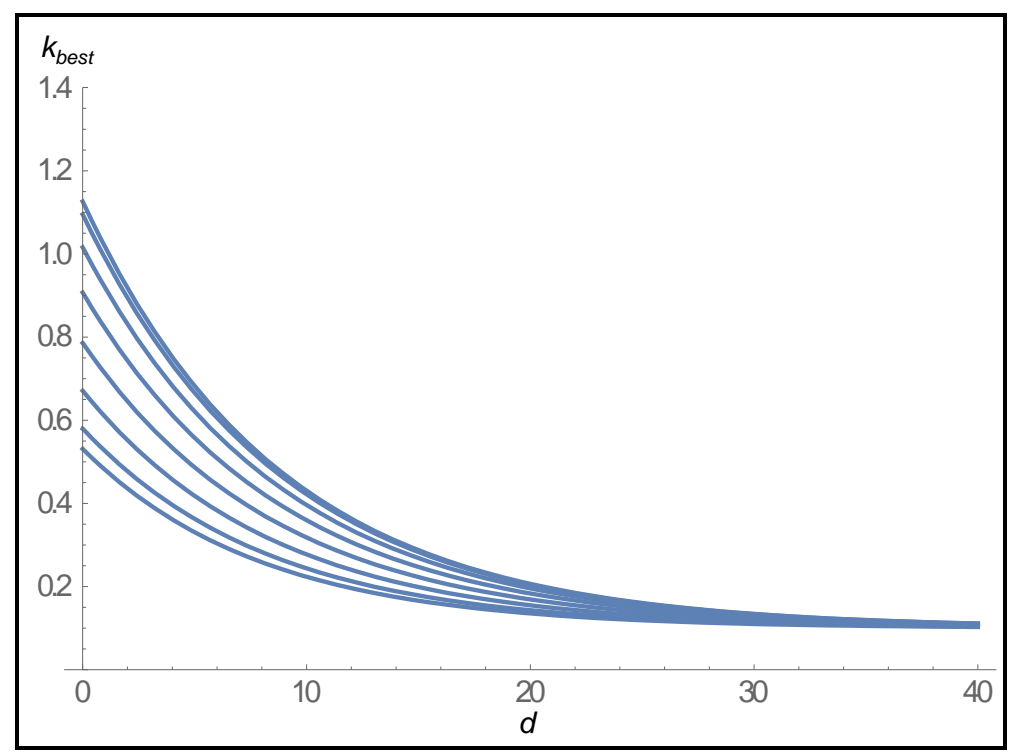

Figure 5. With Equation (7), curves of $k_{\text {best }}$ as functions of separation $d$ for different boundary condition $\varphi_{0}$ are sketched. While in Figure 3, we show $k_{\text {best }}$ vs $d$ only for small $d$, here we show the whole range of $d$ values, from small to large.

Equation (8) then provides the sought sphere-sphere energy-separation curves. The computed results are shown in Figure 6. Based on the shape of the curves, we now can draw conclusions regarding the stability properties predicted by this theory.

\section{Conclusions}

Figure 6 is the main quantitative result of this work and it shows that for all boundary conditions $\varphi_{0}$ the particles attract each other at small separations. This is consistent with all the published experimental literature [19]. In addition, our results show that for large $\varphi_{0}$ the energy decreases monotonically giving rise to repulsion at large separations. While, for small $\varphi_{0}$ there are plateaus that suggest the existence of secondary minima. [15] For all values of $\varphi_{0}$ there are local minima at distances larger than 30, but they cannot be expected to represent experimental behavior since they correspond to distance too large compared to the size of the particles. We also notice that the peak positions of the energy curves shift to larger distances as $\varphi_{0}$ increases, as expected. Finally the behavior of the screening parameter as shown in Figure 5 shows a strong dependence with distance and reducing its value by more than $50 \%$. This has been observed in experimental measurements [15].

For the AFM community, these results are useful in comparing experimental forces with the derivative of the curves in Figure 6. Although the results presented here are limited to particles of the same size, it is clear that the extension of two different radii amounts to adding a second parameter to Equation (6). With that modification, and by choosing the proper theoretical curve, one can infer the charge of the particle interacting with the AFM tip. 


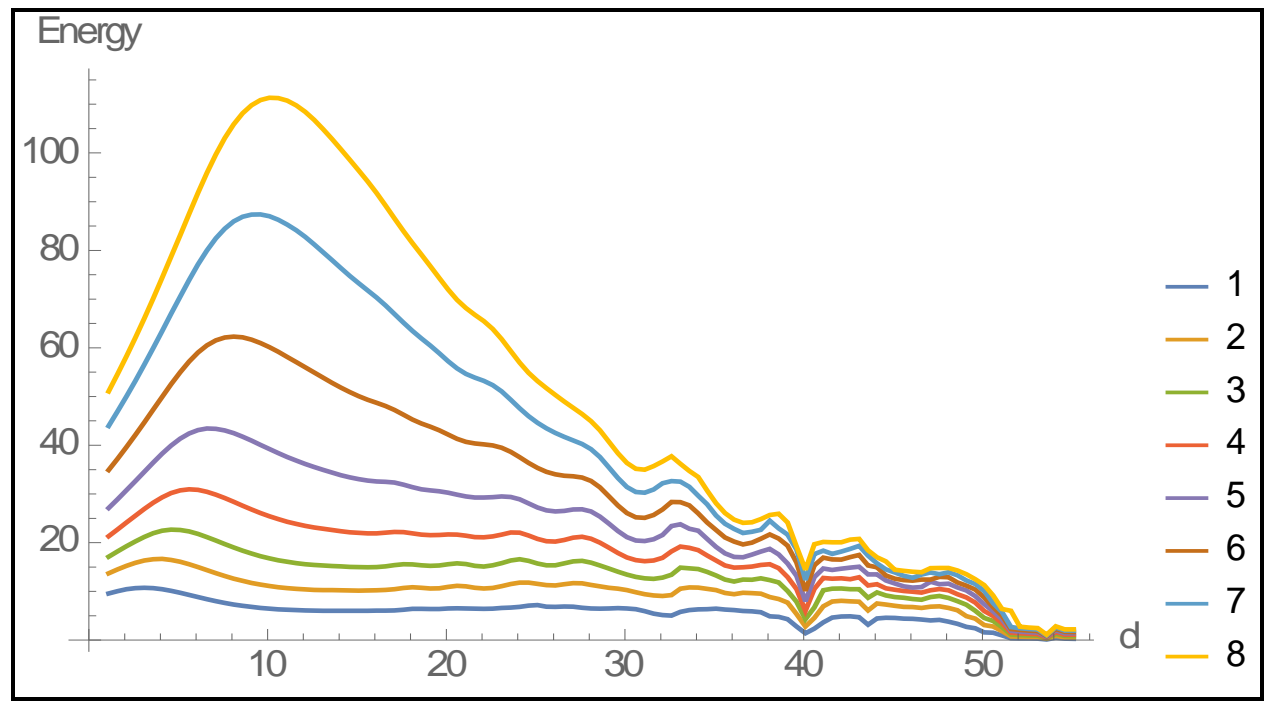

Figure 6. The energy-separation curves for $\varphi_{0}$ from 1 to 8 .

\section{Acknowledgements}

Funding for this project comes from the National Science Foundation grant \#CHE1508085 .

\section{References}

[1] Zypman, F.R. and Eppell, S.J. (2013) Electrostatic Force Curves in Finite-Size-Ion Electrolytes. Langmuir, 29, 11908-11914. http://dx.doi.org/10.1021/la402344m

[2] McLaughlin, S. (1989) The Electrostatic Properties of Membranes. Annual Review of Biophysics and Biophysical Chemistry, 18, 113-136. http://dx.doi.org/10.1146/annurev.bb.18.060189.000553

[3] Maver, U., Velnar, T., Gaberšček, M., Planinšek, O. and Finšgar, M. (2016) Recent Progressive Use of Atomic Force Microscopy in Biomedical Applications. TrAC Trends in Analytical Chemistry, 80, 96-111. http://dx.doi.org/10.1016/j.trac.2016.03.014

[4] Jarmusik, K.E., Eppell, S.J., Lacks, D.J. and Zypman, F.R. (2011) Obtaining Charge Distributions on Geometrically Generic Nanostructures Using Scanning Force Microscopy. Langmuir, 27, 1803-1810. http://dx.doi.org/10.1021/la104153p

[5] Napper, D.H. (1970) Colloid Stability. Product R\&D, 9, 467-477.

[6] Israelachvili, J.N. (1997) Intermolecular and Surface Forces. Academic Press, London.

[7] Birdi, K.S. (2016) Handbook of Surface and Colloid Chemistry. 4th Edition, Chapter 1, CRC Press, Taylor and Francis, London, New York, Boca Raton, 1-144.

[8] Evans, F. and Wennerström, H. (1994) The Colloidal Domain: Where Physics, Chemistry, Biology, and Technology Meet. VCH Publishers, New York.

[9] Verwey, J. and Overbeek, J.Th.G. (1984) Theory of Stability of Lyophobic Colloids. Elsevier Publishing Company, New York, 22-30.

[10] Davis, M.E. and McCammon, J.A. (1990) Electrostatics in Biomolecular Structure and Dynamics. Chemical Reviews, 90, 509-521. http://dx.doi.org/10.1021/cr00101a005

[11] Brenner, S.L. and Roberts, R.E. (1973) Variational Solution of the Poisson-Boltzmann Equation for a Spherical Colloidal Particle. The Journal of Physical Chemistry, 77, 2367-2370. 
http://dx.doi.org/10.1021/j100639a001

[12] Bohinc, K., Iglic, A. and Slivnik, T. (2008) Linearized Poisson Boltzmann Theory in Cylindrical Geometry. Elektrotechniškivestnik, 75, 82-84.

[13] Henderson, D. and Chan, K.Y. (1992) Potential Distribution in the Solution Interface of a Scanning Tunneling Microscope. Journal of Electroanalytical Chemistry, 330, 395-406. http://dx.doi.org/10.1016/0022-0728(92)80320-4

[14] Chan, K.Y., Henderson, D. and Stenger, F. (1994) Nonlinear Poisson-Boltzmann Equation in a Model of a Scanning Tunneling Microscope. Numerical Methods for Partial Differential Equations, 10, 689-702.

[15] Pecina, O., Schmickler, W., Chan, K.Y., Henderson, D.J. (1995) A Model for the Effective Barrier Height Observed with a Scanning Tunneling Microscope. Journal of Electroanalytical Chemistry, 396, 303-307. http://dx.doi.org/10.1016/0022-0728(95)03853-9

[16] Zypman, F. (2006) Exact Expressions for Colloidal Plane-Particle Interaction Forces and Energies with Applications to Atomic Force Microscopy. Journal of Physics: Condensed Matter, 18, 2795-2803. http://dx.doi.org/10.1088/0953-8984/18/10/005

[17] Jackson, D. (1998) Classical Electrodynamics. John Wiley, New York.

[18] Hill, L. (1960) An Introduction to Statistical Thermodynamics. Addison Wesley, Reading.

[19] Israelachvili, J.N. and Adams, G.E. (1978) Measurement of Forces between Two Mica Surfaces in Aqueous Electrolyte Solutions in the Range 0 - $100 \mathrm{~nm}$. Journal of the Chemical Society, Faraday Transactions, 74, 975-1001. http://dx.doi.org/10.1039/f19787400975

\section{Submit or recommend next manuscript to SCIRP and we will provide best service for you:}

Accepting pre-submission inquiries through Email, Facebook, LinkedIn, Twitter, etc. A wide selection of journals (inclusive of 9 subjects, more than 200 journals)

Providing 24-hour high-quality service

User-friendly online submission system

Fair and swift peer-review system

Efficient typesetting and proofreading procedure

Display of the result of downloads and visits, as well as the number of cited articles

Maximum dissemination of your research work

Submit your manuscript at: http://papersubmission.scirp.org/

Or contact jamp@scirp.org 